\title{
Extensão máxima de retalho pediculado de omento maior através de túnel subcutâneo para ossos longos em cães
}

\author{
Maximum length of greater omentum pedicle flap through subcutaneous tunnel for \\ long bones in dogs
}

\author{
Kelly Cristiane Ito ${ }^{{ }^{*}}$ Cássio Ricardo Auada Ferrigno ${ }^{\mathrm{I}}$ Flávio Rocha Alves ${ }^{\mathrm{II}}$
}

\section{RESUMO}

Há tempo sabe-se que o omento humano pode promover atividade angiogênica em estruturas adjacentes nas quais ele é aplicado. Na medicina veterinária, são poucas as pesquisas com retalho pediculado de omento maior como indutor angiogênico e imunogênico, porém suas propriedades de adesão e drenagem são bem conhecidas. Os objetivos deste estudo foram criar um retalho pediculado de omento maior, mensurar seu comprimento durante as etapas de criação $e$ avaliar a possibilidade de alcance para ossos longos (fêmur, tíbia, úmero, rádio e ulna) através de túnel subcutâneo, visando a utilizá-lo futuramente como indutor angiogênico em focos de fratura, para aceleração da osteogênese e controle de infecções ósseas. Foram utilizados 30 cadáveres frescos de cães de todas as raças, com exceção dos condrodistróficos. Os resultados foram conclusivos e confirmaram a possibilidade de alcance do retalho de omento para ossos longos de cadáveres de cães em que todos os retalhos alcançaram a metáfise distal dos ossos avaliados. A média de comprimento do omento, em camada dupla, dos 30 animais avaliados foi de 30,87cm; da camada simples foi de $54,37 \mathrm{~cm}$ e do retalho em $L$ foi de $92,7 \mathrm{~cm}$. Com a extensão máxima do omento, foi possível alcançar as metáfises distais de todos os ossos propostos, com comprimento médio excedente de $29,87 \mathrm{~cm}$ para fêmur, $20,73 \mathrm{~cm}$ para tíbia/fíbula, $25,13 \mathrm{~cm}$ para úmero e $16,27 \mathrm{~cm}$ para rádio/ ulna. As variáveis peso e retalho em $L$ avaliadas estatisticamente de cada indivíduo apresentaram correlação positiva moderada. Concluiu-se que, em cadáveres de cães, é possível levar o retalho pediculado de omento maior através de túnel subcutâneo para metáfise distal de ossos longos e que, quanto maior o peso do animal, maior o comprimento do retalho em $L$.

Palavras-chave: indutor angiogênico, tunelização, canino, omentalização.

\section{ABSTRACT}

It's known for long time hat the human omento can promote angiogenic activity in adjacent structures in which it is applied. In veterinary medicine, there is little research with greater omentum flap as angiogenic and immunogenic inductor, however, their adhesion properties and drainage are well known. This study wondered whether if the greater omentum can be used as angiogenic inductor in bone fractures, and therefore increase osteogenenic rates and decreasing bone infection. Initially it was designed an experimental study which aimed to obtain a greater omentum pedicle flap and conduct it so long as possible through a subcutaneous defect in order to reach long bones (femur, tibia, humerus, radius/ulna). For the experiment it was used 30 dogs cadavers of all breeds, except condrosdistrophics breeds. The results were conclusive and confirmed the possibility of reaching the omentum flap for long bones. All the animals had reached the distal half of the evaluated bones. Results were satisfactorily accomplished and in $100 \%$ of the cases the flap reached the distal half of the evaluated bones. The averages of the different flap length were: $30.87 \mathrm{~cm}$ when double layer was used; $54.37 \mathrm{~cm}$ in simple layer; and $92.7 \mathrm{~cm}$ when the flap was built in $L$. The maximum length of the omentum has secured the possibility of reaching the distal metaphases of all the bones studied. The average length exceeded $29.87 \mathrm{~cm}$ to femur, $20.73 \mathrm{~cm}$ to tibia/fibula, $25.13 \mathrm{~cm}$ to humerus, and $16.27 \mathrm{~cm}$ to radius/ulna. The flap length variety statistically evaluated showed moderate positive correlation on the presented individuals. It was concluded that the omentum pedicle flap can be taken through the subcutaneous defect until the distal metaphase of the long bones of dogs cadavers without tension. Animals with high corporal weight have the biggest L flap length.

Key words: angiogenic inducer, tunnelling, canine, omentalization.

IFaculdade de Medicina Veterinária e Zootecnia (FMVZ), Universidade de São Paulo (USP). Endereço para correspondência: Rua José Alves Cunha Lima, 159, ap 51, bl 1, 05360-050, Butantã, SP, Brasil. E-mail: kellyito@usp.br.

"Ouro fino Saúde Animal, Belo Horizonte, MG, Brasil. 


\section{INTRODUÇÃO}

A palavra omento deriva de ancestrais egípcios, que, quando embalsamavam corpos humanos, usavam-no para avaliar o presságio da alma olhando as variações de forma daquele que hoje conhecemos como omento. A visão convencional sobre o omento é que representa um papel central na defesa peritoneal por aderir-se em focos de inflamação, absorvendo bactérias e outros contaminantes, e fornece leucócitos para reação imunológica local (PLATELL et al., 2000).

$\mathrm{Na}$ ortopedia veterinária, o alto índice de fraturas ósseas, suas complicações operatórias e pósoperatórias e o longo tempo de consolidação levam à busca de fatores que acelerem esse processo. De acordo com o grau de traumatismo e a complexidade da fratura, ocorre maior ou menor interrupção do fluxo sanguíneo para o osso, comprometendo a fisiologia normal e, consequentemente, sua resposta osteogênica na formação de calo ósseo (PIERMATTEI \& FLO 1999).

GOLDSMITH (1967) realizou trabalho experimental em cães no qual levou omento para extremidades superior (tríceps) e inferior (quadríceps), por meio de túnel subcutâneo, e comprovou patência vascular do pedículo 30 dias após a tunelizacão. A partir desses achados, foi realizada tunelizacão subcutânea em pacientes humanos portadores de linfedema crônico para membro superior e membro inferior, sendo obtido um retalho funcional após tunelização. O mesmo autor, em 1973, obteve sucesso com tunelização via subcutânea, utilizando retalho pediculado de omento para superfície cerebral, e comprovou a capacidade de angiogênese desse órgão, além de comprovar que a passagem através de túnel subcutâneo é possível sem efeitos deletérios ao pedículo.

Caso se comprove a possibilidade de alcance do omento para ossos longos, tal descoberta seria de grande valia para posteriores pesquisas em ortopedia veterinária, principalmente em casos de não união, aceleração de cicatrização óssea e osteomielites. Diante disso, o objetivo deste trabalho foi avaliar o modelo de retalho pediculado de omento maior, o seu comprimento, o seu trajeto e a sua possibilidade de alcance para ossos longos de cães, baseando-se na hipótese de que o omento aumenta o suprimento vascular de tecidos e melhora a função imune.

\section{MATERIAL E MÉTODOS}

Para o experimento, foram utilizados 30 cadáveres frescos de cães, com massa corpórea de 13 a 26kg, sendo 10 fêmeas e 20 machos, sem raça definida, distribuídos aleatoriamente em dois grupos de 15 animais. O grupo I foi submetido à tunelização para fêmur e tíbia/fíbula, e o grupo II foi submetido à tunelização para úmero e rádio/ulna. Foram excluídos da pesquisa cães condrodistróficos, com sinais de intervenção cirúrgica abdominal, aderências abdominais ou com quaisquer alterações anatômicas do omento maior.

Para a confecção do retalho de omento maior, foram realizadas celiotomia mediana pré-retroumbilical e exteriorização do baço, estômago e omento em camada dupla (Figura 1 A). A camada omental dorsal foi desinserida do pâncreas e estendida caudalmente, prolongando o omento e formando o retalho em camada simples (Figura 1 B). A incisão em forma de L-invertido foi realizada iniciando pelo lado direito abaixo do ligamento gastroesplênico transversalmente no omento. Enquanto o omento foi incisado até um terço de sua largura, os vasos omentais foram seccionados e ligados. A incisão prosseguiu caudalmente paralela aos vasos omentais, e a incisão longitudinal se estendeu ao longo de dois terços do comprimento do omento. O lado direito do omento foi então rotacionado caudalmente $180^{\circ}$ até extensão completa do pedículo (Figura 1 C).

O comprimento do omento maior foi mensurado a cada estágio de confecção do retalho pediculado com auxílio de uma fita métrica. Os pontos de medida das camadas foram a margem da curvatura maior do estômago e a margem do omento estendido, com a fita métrica posicionada perpendicular ao estômago e paralela ao eixo longitudinal do corpo do animal. A mensuração do comprimento sobressalente de omento foi realizada após o alcance à metáfise distal de todos os ossos avaliados. Destaca-se que o mesmo retalho utilizado para avaliar alcance ao fêmur foi utilizado para tíbia/fíbula, e o retalho utilizado para úmero foi o mesmo utilizado para o rádio/ulna.

O trajeto do túnel subcutâneo foi feito por divulsão e descolamento do subcutâneo da musculatura e sua largura de aproximadamente cinco centímetros em todos os animais. Este teve início no bordo caudal da incisão da cicatriz umbilical, percorrendo o músculo reto do abdome em direção caudal. Na região inguinal direita, foi feita uma incisão de pele de três centímetros para facilitar a confecção do restante do trajeto do túnel. A partir da incisão cutânea na região inguinal, o túnel seguiu passando pela região cranial do músculo quadríceps e percorreu trajeto paralelo ao nervo femoral cutâneo sobre os músculos sartório, tensor da fáscia lata e vasto lateral, até o acesso ao fêmur. 


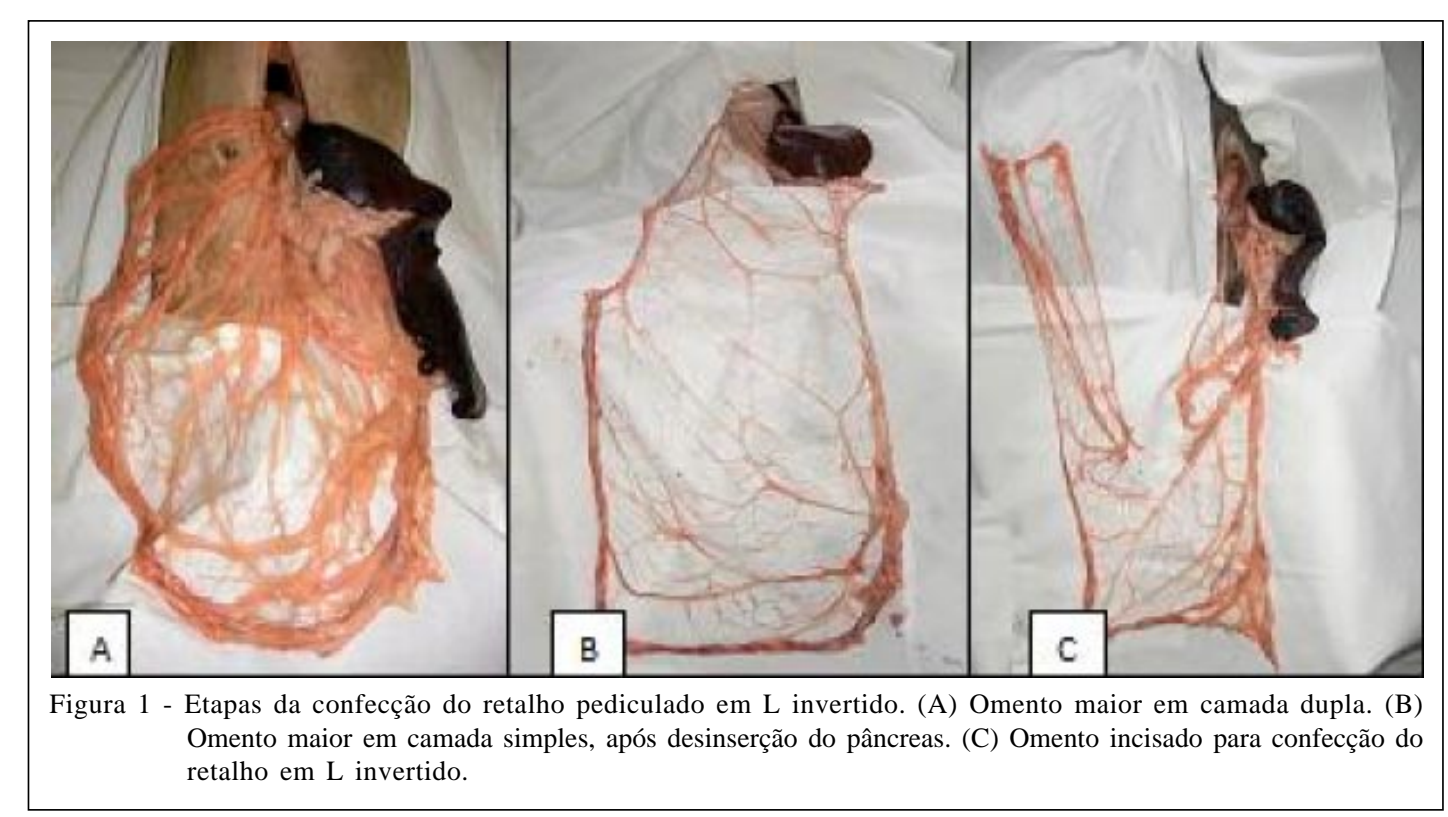

Após confecção do túnel e a confecção do acesso à metáfise distal do fêmur, foi realizada delicadamente a passagem do retalho através do túnel, com auxílio dos dedos e de uma pinça Kelly, mantendoo úmido para facilitar a passagem sem resistência e sem rompimento do omento até a metáfise distal do fêmur.

O túnel para a tíbia foi o mesmo feito para o fêmur até a incisão na região inguinal. A partir da incisão na região inguinal, o túnel seguiu antero-medial ao músculo quadríceps, paralelo à artéria e veia femoral e artéria e veia safena até a tíbia. Depois de confeccionados o túnel e o acesso à metáfise distal da tíbia, foi realizada delicadamente a passagem do retalho de omento por meio do túnel subcutâneo até a metáfise distal da tíbia. A tunelização para úmero iniciou-se na borda cranial da celiotomia pré-retroumbilical. A divulsão entre a pele e o subcutâneo foi realizada sobre o esterno, passando pelo processo xifoide até a inserção do músculo peitoral superficial porção descendente no esterno. Uma incisão cutânea de três centímetros foi feita na região da cartilagem xifoide para facilitar a passagem do omento. O túnel avançou em sentido transversal da altura do terço médio desse músculo, passando sobre o tubérculo maior do úmero direito e sobre o músculo deltoide até alcançar o úmero. Depois de confeccionados o túnel e o acesso para o úmero distal, o retalho foi umedecido com solução fisiológica e cuidadosamente passado através do túnel subcutâneo até a metáfise distal do úmero. A tunelização para rádio e ulna foi a mesma feita para úmero até a região da incisão cutânea na cartilagem xifoide. A partir daí o túnel seguiu sobre o músculo peitoral superficial até sua inserção no úmero medial e seguiu medialmente ao úmero, paralelo à artéria e veia braquial até o rádio. Após a finalização do acesso e túnel, foi feita delicadamente a passagem do retalho umedecido em solução fisiológica através do túnel subcutâneo até a incisão cutânea na região de xifoide e posteriormente até a metáfise distal do rádio e da ulna.

Os resultados foram analisados por meio do programa computacional Statistical Analysis System (SAS 2001), sendo anteriormente verificada a normalidade dos resíduos pelo Teste de Shapiro-Wilk (Proc. Univariate), e a homogeneidade das variâncias comparada pelo Teste de Hartley (Ott 1993). Todos os dados variáveis dependente atenderam a essas premissas. Os dados dos comprimentos das camadas (dupla e simples) e do retalho em $L$ foram submetidos à análise de variância, que separou como causa de variação o efeito de tratamento. O efeito de tratamento foi separado por meio do teste de média Tukey. Tal análise foi realizada utilizando-se o procedimento General Linear Model (Proc. GLM do SAS). Além disso, os dados foram analisados por correlação linear para obter o valor de r (rho) e a probabilidade entre os dados obtidos neste trabalho. Como todos os dados possuem distribuição normal e homogeneidade das variâncias, utilizou-se a correlação de Pearson (Proc. Corr. do SAS). Foi utilizado o nível de significância de 5\% para os testes realizados, com exceção do teste de Tukey, para o qual se utilizou o nível de significância de $1 \%$.

Ciência Rural, v.40, n.3, mar, 2010. 


\section{RESULTADOS}

Na tabela 1, estão os valores médios das sequências da técnica de incisão para obtenção do retalho em $\mathrm{L}$, suas respectivas médias, o desvio padrão (DP) e as probabilidades.

O teste de correlação aplicado entre peso e comprimento final do retalho (retalho em L) total e nos grupos (fêmeas, machos, retalho para membro anterior e retalho para membro posterior) demonstrou significância estatística, como demonstra a tabela 2. As variáveis avaliadas apresentaram correlação positiva moderada em todos os casos, ou seja, quanto maior o peso, maior o comprimento do retalho em $\mathrm{L}$ dos animais avaliados em cada grupo.

\section{DISCUSSÃO}

ALDAY \& GOLDSMITH(1972) descreveram técnicas para prolongamento de omento maior com base no estudo da anatomia vascular do órgão em humanos. Observaram cinco variações no suprimento vascular e descreveram cinco técnicas para prolongamento de cada uma. Os procedimentos de extensão utilizados em humanos foram adaptados para uso em modelos animais e estudos clínicos em cães. Há muitas diferenças anatômicas entre o omento humano e canino, envolvendo o suprimento vascular e a anatomia. Segundo ROSS \& PARDO (1993), o omento canino recebe a maioria do suprimento sanguíneo dos vasos que surgem da arcada gastroepiploica direita e artéria esplênica esquerda. Baseados nessa arquitetura vascular, estes descreveram a primeira técnica para prolongamento do omento em cães, a mesma utilizada neste trabalho, uma vez que desenvolveram uma técnica simples para criação do retalho e comprovaram a manutenção da integridade vascular do pedículo.

Este estudo foi realizado em cadáveres de cães, e a integridade da vascularização do retalho pediculado em L-invertido não foi avaliada, no entanto,
ROSS \& PARDO (1993) comprovaram que a patência vascular do retalho pediculado em L-invertido em cães é mantida, confeccionado por meio da mesma técnica utilizada neste trabalho.A tunelização de retalho de omento pediculado em humanos tem seu uso restrito, uma vez que DAS (1976) demonstrou, em trabalho com 200 cadáveres humanos, que é possível alcançar caixa torácica, joelhos e cotovelos com a extensão do omento, porém somente 15\% alcançaram o tornozelo e 5\% o dedão do pé. Em humanos, a utilização do enxerto de omento para extremidades distais é realizada por meio de cirurgias de microanastomoses devido a essa restrição de alcance. O presente trabalho demonstra que em cães essa restrição não existe, uma vez que foi possível alcançar a metáfise distal de rádio/ulna e tíbia/ fíbula com sobressalente em todos os animais avaliados. Acredita-se que não haja essa restrição em cães em razão da estrutura quadrupedal do cão.

Os animais utilizados neste experimento não haviam sofrido cirurgia abdominal prévia para não interferir no comprimento final do omento; porém, LOPEZ et al. (1998), em estudo comparativo em humanos com retalho de omento e retalho do músculo peitoral maior para tratamento de mediastinite após cirurgia cardíaca aberta, relataram não ter encontrado dificuldades no prolongamento do retalho e no alcance ao seu destino em pacientes que haviam sofrido intervenções cirúrgicas abdominais prévias, pois o prolongamento pode ser feito, tanto baseado na artéria gastroepiploica direita, quanto nos vasos contralaterais.

Segundo LOPEZ et al. (1998), o uso do omento tem algumas desvantagens, pelo fato de ser um procedimento cirúrgico que requer laparotomia e por comunicar uma cavidade estéril com uma não estéril, além de comprometer a extensão do omento em casos de pacientes em que foram realizadas cirurgias abdominais prévias. O omento maior é um órgão fino e delicado. A manipulação durante o experimento foi feita cuidadosamente, e o órgão foi mantido umedecido constantemente com solução fisiológica. Todo cuidado

Tabela 1 - Valores médios das sequências da técnica de incisão para a obtenção do retalho em L, suas respectivas médias, seu desvio padrão (DP) e suas probabilidades.

\begin{tabular}{|c|c|c|c|c|c|c|}
\hline & Camada dupla & Camada simples & Flap L & Média & DP & Probabilidade \\
\hline Úmero - Rádio & $32,29^{c}$ & $58,36^{\mathrm{b}}$ & $97,14^{\mathrm{a}}$ & 63,18 & 29,16 & $<0,0001$ \\
\hline Fêmur - Tíbia & $28,29^{c}$ & $50,36^{\mathrm{b}}$ & $88,29^{\mathrm{a}}$ & 55,44 & 25,48 & $<0,0001$ \\
\hline Total & $30,87^{\mathrm{c}}$ & $54,37^{\mathrm{b}}$ & $92,70^{\mathrm{a}}$ & 59,31 & 27,50 & $<0,0001$ \\
\hline Macho & $31,32^{\mathrm{c}}$ & $55,10^{\mathrm{b}}$ & $95,85^{\mathrm{a}}$ & 56,07 & 25,67 & $<0,0001$ \\
\hline Fêmea & $28,11^{\mathrm{c}}$ & $52,90^{\mathrm{b}}$ & $86,40^{\mathrm{a}}$ & 60,93 & 28,44 & $<0,0001$ \\
\hline
\end{tabular}

${ }^{\mathrm{a}, \mathrm{b}}$ Letras sobrescritas diferentes diferem no teste de Tukey $(\mathrm{P}<0,001)$. 
Tabela 2 - Coeficientes de correlação (r) e probabilidades entre as variáveis respostas peso - Flap L em diferentes grupos.

\begin{tabular}{lll}
\hline Peso - Flap L (úmero - rádio) & 0,6223 & 0,0132 \\
\hline Peso - Flap L (fêmur - tíbia) & 0,5776 & 0,0241 \\
Peso - Flap L (total) & 0,5733 & 0,0009 \\
Peso - Flap L (fêmea) & 0,6894 & 0,0274 \\
Peso - Flap L (macho) & 0,4259 & 0,0611 \\
\hline
\end{tabular}

durante a manipulação e tunelização foi tomado para que não houvesse ruptura e o comprimento final não fosse comprometido.

ROSS \& PARDO, (1993) realizaram prolongamento de omento em duas etapas com desinserção do pâncreas e retalho em L invertido. Somente com a extensão dorsal foi verificada possibilidade de alcance a regiões torácica, abdominal e inguinal. Com a extensão completa, o pedículo omental alcançou todas as partes do corpo sem remover o pedículo vascular. Entretanto, a avaliação feita por esses autores foi realizada sobre a pele por fora do corpo. No presente trabalho, essa avaliação foi feita através de tunelização com trajeto específico e confirmou os achados de ROSS \& PARDO (1993), os quais obtiveram alcance do omento na metáfise distal de rádio/ulna e tíbia/fíbula em todos os animais avaliados.
Devido ao grau de complexidade e às complicações cirúrgicas inerentes a confecção do retalho, a importância deste trabalho foi fornecer o tamanho aproximado do omento esperado em um paciente canino, a partir do seu peso, e determinar se este alcança a área pretendida previamente à laparotomia, como demonstra a representaçao grafica de correlaçao entre peso e comprimento do flap em $\mathrm{L}$ dos grupos avaliados na figura 2.

\section{CONCLUSÕES}

A partir dos resultados obtidos, concluiuse que é possível alcançar a metáfise distal dos ossos longos fêmur, tíbia/fíbula, úmero e rádio/ulna de cadáveres de cães, a partir da extensão do omento maior em retalho em $\mathrm{L}$ invertido e mantendo seu pedículo vascular.

O comprimento final do retalho pediculado é variável e diretamente proporcional ao peso do animal, independente do sexo; e quanto maior o peso do animal, maior o comprimento do retalho de omento.

\section{COMITÊ DE ÉTICA}

O estudo e a metodologia empregada foram aprovados pela Comissão de Ética da Universidade de São Paulo, protocolado sob o $\mathrm{n}^{\mathrm{0}}$ 1043/2007.

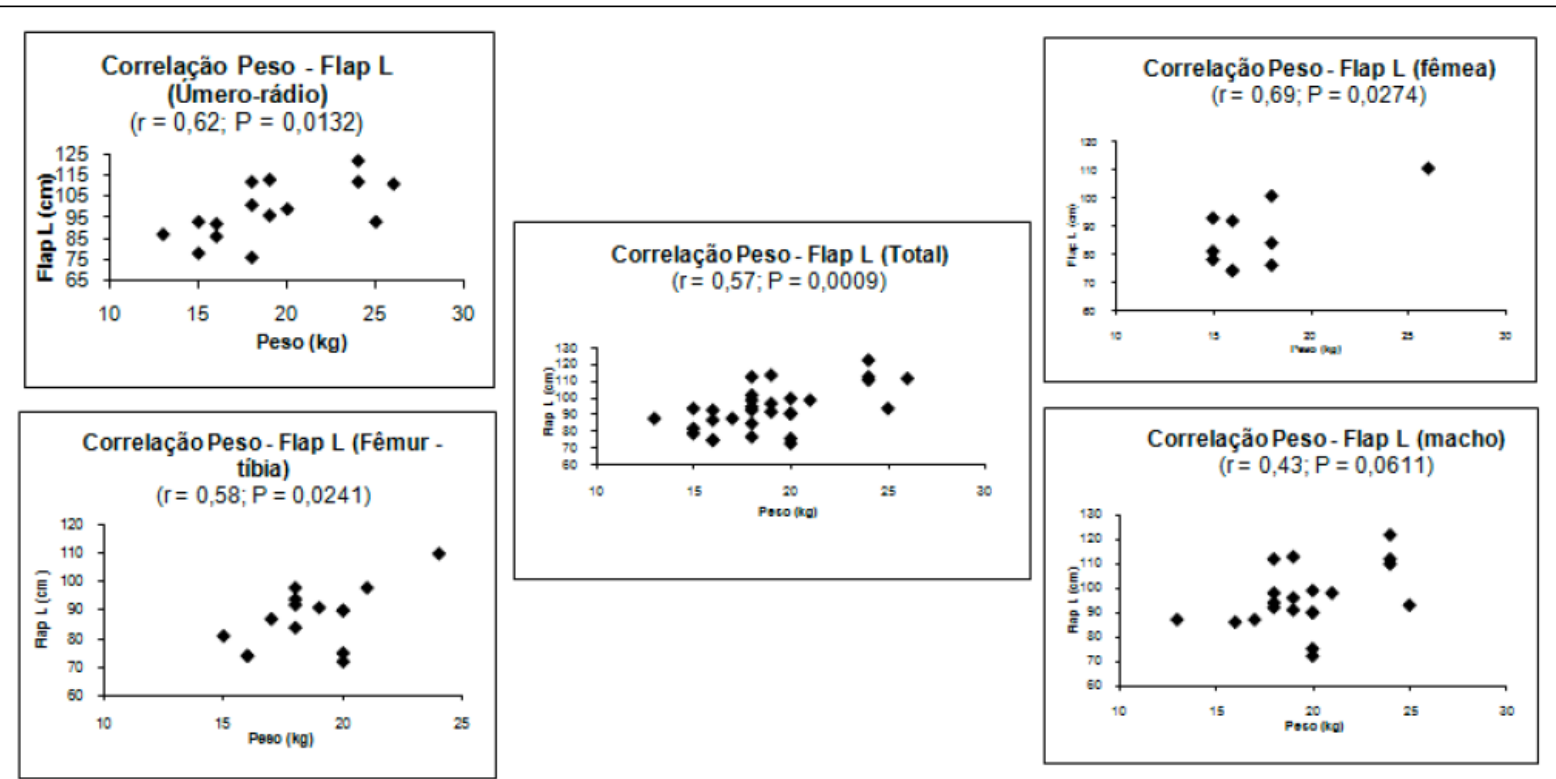

Figura 2 - Representação gráfica individual dos coeficientes de correlação (r) e probabilidades entre as variáveis respostas peso Flap L dos grupos de animais submetidos à tuneliação para membro torácico, membro pélvico, fêmeas e machos e o total de animais avaliados. 


\section{REFERÊNCIAS}

ALDAY, E.S.; GOLDSMITH, H.S.. Surgical technique for omental lengthening based on arterial anatomy. Surgery Gynecology \& Obstetrics, v.135, p.103-107, 1972.

DAS, S.K. The size of the human omentum and methods of lengthening it for transplantation. Brazilian Journal of Plastic Surgery, v.29, p.170-174, 1976.

GOLDSMITH, H.S. et al. Brain vascularization by intact omentum. Archives of Surgery, v.106, p.695-698, 1973.

GOLDSMITH, H.S. et al. Relief chronic lymphedema by omental transposition. Annals of Surgery, v.166, n.4, p.573583, 1967. Disponível em: http://www.ncbi.nlm.nih.gov/pmc/ articles/PMC1477440/pdf/annsurg00442-0065.pdf. Acesso em: 17 out. 2009

LOPEZ, M.H. et al. Omentum flap versus pectoralis major flap in the treatment of mediastinitis. Plastic and Reconstructive Surgery, v.101, n.6, p.1481-1485, 1998. Disponível em: http:/ovidsp.tx.ovid.com/spb/ ovidweb.cgi?QS2=434f4e1a73d37e8c8b3eab7e2fc8fd7c7f28bae3b1a509 ef3a6556a13eb8ff865e6cdb372a7c426de24b533586fd50bc913a47 e72d2b2d1d857377f6bbe8d531ea81bee0c20d9eda65d30 0139cabbfb5c760cbfe068e8dc2e015b18e0551a2dd0791 8ecd5854c0cae06e7aec5fefc727a772db51ffd5026aea86510ed 1ab9954343da4c97bd21c88fa3f4ffbfa17e7d9787e38ce8db8e7 c $161916 \mathrm{ff} 3132056$ a 0 c ba 131747 d 9 b c 85 fd 43154 b 6 e 242 a 9 c d f 61828 b 67 e f 854303 e 93 a 4 846019 c 1 f 9505 a 988 a 7321 d a 67 b 503 e 508 c 190 d d a 29 a b 1 c 2 f 17 e f 60110 c 11 e

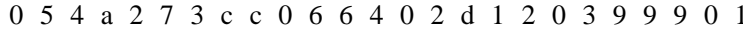
07888f2f7ed128714c9506f138af9cda028. Acesso em: 17 out. 2009.

PLATELL, C. et al. The omentum. World Journal of Gastroenterology, v.6, n.2, p.169-176, 2000. Disponível em: http://www.wjgnet.com/1007-9327/6/169.pdf. Acesso em 17 out. 2009

PIERMATTEI, D.L.; FLO, G.L. Fraturas: classificação, diagnóstico e tratamento. In: __. Manual de ortopedia e tratamento das fraturas dos pequenos animais. 3.ed. São Paulo: Manole, 1999. p.24-138.

ROSS, W.E.;PARDO, A.D. Evaluation of an omental pedicle extension technique in the dog. Veterinary Surgery, v.22, n.1, p.37-43, 1993.

SAS Institute. SAS user's guide: statistics. 5.ed. Cary, NC, 2001. 285p. 